Drug Diffusion, Integration and Stability of Nano-Engineered Drug-Releasing Implants in

\title{
Bone Ex-Vivo
}

Shafiur Rahman ${ }^{1 *}$, Karan Gulati ${ }^{1 *}$, Masakazu Kogawa ${ }^{2}$, Gerald J. Atkins ${ }^{2}$, Peter Pivonka ${ }^{3}$,

David M. Findlay ${ }^{2 凶}$, Dusan Losic ${ }^{1 凶}$

(

${ }^{1}$ School of Chemical Engineering, University of Adelaide, SA, Australia

${ }^{2}$ Discipline of Orthopaedics \& Trauma, University of Adelaide, SA, Australia

${ }^{3}$ Australian Institute for Musculoskeletal Science, University of Melbourne, VIC, Australia

*Equal first author status

Corresponding Author:

Prof. Dusan Losic

School of Chemical Engineering, University of Adelaide, SA 5005, Australia

Phone: +61 88013 4648, Email: dusan.losic@adelaide.edu.au

Prof. David M. Findlay

Discipline of Orthopaedics and Trauma, University of Adelaide, SA 5005, Australia

Phone: +61 88222 5621, Email: david.findlay@adelaide.edu.au

Keywords: local drug delivery, bone therapy, titania nanotubes, drug-releasing implants, drug

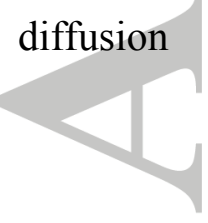

This article has been accepted for publication and undergone full peer review but has not been through the copyediting, typesetting, pagination and proofreading process which may lead to differences between this version and the Version of Record. Please cite this article as an 'Accepted Article', doi: 10.1002/jbm.a.35595 


\begin{abstract}
To treat skeletal conditions such as bone infections, osteoporotic fractures and osteosarcoma, it would be ideal to introduce drugs directly to the affected site. Localized drug delivery from the bone implants is a promising alternative to systemic drug administration. In this study we investigated electrochemically nano-engineered Ti wire implants with titania nanotubes (TNTs), as minimally invasive drug-releasing implants for the delivery of drugs directly into the bone tissue. Since trabecular bone in vivo contains a highly interconnected bone marrow, we sought to determine the influence of marrow on drug release and diffusion. Electrochemical anodization of Ti wires (length $10 \mathrm{~mm}$ ) was performed to create an oxide layer with TNTs on the surface, followed by loading with a fluorescent model drug, Rhodamine B (RhB). Cores of bovine trabecular bone were generated from the sternum of a young steer, and were processed to have an intact bone marrow, or the marrow was removed. RhB-loaded TNTs/Ti wires were inserted into the bone cores, which were then cultured ex vivo using the ZetOS ${ }^{\mathrm{TM}}$ bioreactor system to maintain bone viability. Release and diffusion of RhB inside the bone was monitored using fluorescence imaging and different patterns of drug transport in the presence or absence of marrow were observed. Scanning electron microscopy of the implants after retrieval from bone cores confirmed survival of the TNTs structures. Histological investigation showed the presence of bone cells adherent on the implants. This study shows a potential of Ti drug-releasing implants based on TNTs technology towards localized bone therapy.
\end{abstract}

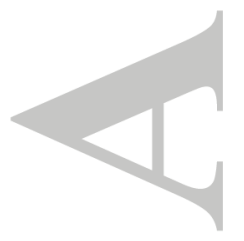

John Wiley \& Sons, Inc. 


\section{INTRODUCTION}

Systemic administration of potential therapeutics to target bone conditions such as infection, cancer or fracture, may be suboptimal in delivering sufficient and sustained agent to the affected site. In addition, systemic delivery exposes the entire body to these agents. Ideally, drugs would be applied locally to affected sites in bone, and as a result many local drug delivery (LDD) solutions have been suggested. ${ }^{1-2}$ This concept has led to the design and development of novel titanium implants that enable prolonged LDD, directly inside/adjacent to the diseased bone tissue. ${ }^{3-4}$ While other bone implant therapeutic solutions have been investigated, such as biopolymer scaffolds and bone cements, these are limited by a lack of mechanical stability and/or unpredictable drug release kinetics. ${ }^{5}$ Hence there is need to modify titanium bone implants in way that controlled and local therapy could be achieved, while maintaining mechanical stability to survive load-bearing conditions.

As a result a number of strategies have been devised to change the surface topography of Ti implants to incorporate drug, for example using conjugated biopolymer coatings. ${ }^{6}$ Micro- or nano-roughness of Ti implants has also been suggested as a means of coating an implant surface with drug, but this approach is problematic due to a lack of control over the release kinetics. ${ }^{3,7-9}$ Alternatively, electrochemical fabrication of nano-porous or nano-tubular structures onto Ti and its alloys has emerged as a viable way to address the abovementioned challenges. ${ }^{2-4}$ These nanoscale porous/tubular structures allow for easy/stable modification of the implants and enable substantial drug loading amounts, controlled release, and enhanced bioactivity required for upregulating bone cell functions. ${ }^{9-11}$ Using electrochemical anodization (EA), which is scalable technology, titania $\left(\mathrm{TiO}_{2}\right)$ nanotubes (TNTs), can be fabricated onto Ti implants with good control over their dimensions and properties. ${ }^{10}$ The application of TNT modified implants for 
loading broad range of therapeutics including antibiotics, anti-inflammatory drugs, growth factors, proteins, anti-cancer drugs, genes, drug carriers as polymer micelles and nanoparticles archiving an enhanced local therapeutic action is confirmed and reported in numerous in-vitro and in-vivo studies. ${ }^{3-6,11-13}$

More recently, TNT fabrication technology has been extended to complex substrate shapes and morphologies, such as Ti wires and pins, in an attempt to target complex bone conditions, including infection and cancer. ${ }^{14-16}$ TNTs/Ti wires loaded with active therapeutics were proposed for in-bone therapy, releasing drug in a three-dimensional fashion from the surface of the minimally-invasive implants. ${ }^{16}$ Our group has previously demonstrated the use of such nano-engineered $\mathrm{Ti}$ wires for delivering therapeutics directly inside bone tissue, using a bovine trabecular bone model ex-vivo. ${ }^{15}$ That proof-of-principle study showed that TNTs/Ti wire implants could release their payload (fluorescent dye) directly inside the bone in the absence of bone marrow, and this could be monitored in situ using fluorescence (FL) imaging. ${ }^{15}$ However, the trabecular bone microenvironment is complex and contains an interconnected bone marrow, consisting of connective tissue, mesenchymal cells, haemopoietic cells and adipocytes, which is likely to influence drug distribution from the implant surface.

We have therefore extended this study to investigate drug-releasing TNTs/Ti wires implanted into bovine trabecular bone cores, comparing marrow free bone with bone cores containing marrow, to replicate the in vivo situation. An optimized anodization procedure was used to fabricate robust TNTs on Ti wires, followed by drug loading and placement inside the trabecular bone core ex-vivo. The proposed experiment is schematically represented in Figure 1. Three experimental setups were considered: bone cores with no marrow (NoBM), bone cores with marrow $(\mathrm{BM})$, and bone cores with marrow treated with heparin $(\mathrm{BM}+\mathrm{HEP})$, the latter to 
determine the effect of anti-coagulant on drug diffusion into bone. The interaction of the bone tissue with the implant was also studied after termination of the dye diffusion study, to determine implant stability post-retrieval. Our results suggest the feasibility of minimally invasive TNTs/Ti wire implants for the local delivery of potent therapeutics into trabecular bone.

\section{MATERIALS AND METHODS}

\section{Materials}

Ti (99.99\%) wires (diameter $0.8 \mathrm{~mm}$ ) were supplied by Nilaco (Japan). Ethylene glycol, lactic acid, acetone, ammonium fluoride, Rhodamine $\mathrm{B}(\mathrm{RhB})$ and heparin were obtained from SigmaAldrich Pty Ltd (Sydney, Australia) and used without further purification. High-purity ultragrade Milli-Q ${ }^{\circledR}$ water (18.2 $\mathrm{M} \Omega \mathrm{cm}$ resistivity) (EMD Millipore Corporation, MA) with additional filtration $(0.22 \mu \mathrm{m})$ was used for the preparation of all reagents.

\section{Fabrication of titania nanotubes on Ti wire implants}

Ti wires were mechanically polished using sand paper, followed by sonication for 30 min in ethanol/acetone. Later, electropolishing was performed in perchloric acid electrolyte (with butanol and ethanol, $\mathrm{P}: \mathrm{B}: \mathrm{E}=1: 6: 9)$ maintained at $4^{\circ} \mathrm{C}$, using $20 \mathrm{~V}$ for $3 \mathrm{~min}$, with a stainless steel counter electrode. The polished wires were cleaned by sonication in ethanol/acetone and dried in $\mathrm{N}_{2}$. The wires were masked using pipette tips, so as to expose a $9 \mathrm{~mm}$ length for anodization. Electrochemical anodization was performed using ethylene glycol electrolyte [1.5 $\mathrm{M}$ lactic acid, $0.1 \mathrm{M}$ ammonium fluoride, $2.5 \%(\mathrm{v} / \mathrm{v})$ water], maintained at $60^{\circ} \mathrm{C}$. A constant voltage of $60 \mathrm{~V}$ was applied for $20 \mathrm{~min}$ with a microprocessor-controlled power supply 
(Agilent). The anodized implants were then rinsed in water, dried in $\mathrm{N}_{2}$ and cut in $10 \mathrm{~mm}$ lengths (with the anodized portion $=9 \mathrm{~mm}$ ).

\section{Morphological characterization of TNTs/Ti wire implants}

Morphological characterization of the TNTs/Ti wires was carried out using field emission scanning electron microscope (FE-SEM) (Quanta 450, The Netherlands). Prior to imaging, samples were mounted on a SEM holder using double-sided conductive tape, and coated with a 5 $\mathrm{nm}$ thick layer of platinum. Images with a range of scan sizes at normal incidence and at a $30^{\circ}$ angle were acquired from the top/bottom surfaces and cross-sections.

\section{Drug loading and in vitro drug release}

TNTs/Ti wire implants were immersed in $\mathrm{RhB}$ solution $(50 \mathrm{mg} / \mathrm{ml}$ in water), sonicated for $5 \mathrm{~min}$ and then allowed to soak for $2 \mathrm{~h}$ with gentle shaking every $30 \mathrm{~min}$ to promote drug loading. Later the drug loaded implants were dried in air and kept under vacuum for $2 \mathrm{~h}$, followed by gently removing any excess drug from the implant surfaces using a soft tissue wet with phosphate buffered saline (PBS, pH 7.4). To quantify the loading amount, the dye loaded implants were heated in TGA (thermo-gravimetric analysis, TA Instruments, Q500) furnace to $500^{\circ} \mathrm{C}$ at the rate of $10^{\circ} \mathrm{C} / \mathrm{min}$, followed by analysis of the weight change corresponding to the decomposition range of $\mathrm{RhB}$.

Later the RhB implants were sterilized using low-temperature hydrogen peroxide plasma (Sterrad ${ }, 100 \mathrm{NX}^{\mathrm{TM}}$ System, Advanced Sterilization Products, USA). For in vitro drug release, RhB-loaded TNTs/Ti wires were immersed in $5 \mathrm{ml} \mathrm{PBS} \mathrm{(pH} \mathrm{7.4)} \mathrm{at} 25^{\circ} \mathrm{C} .3 \mathrm{ml}$ aliquots of PBS were drawn (and immediately replaced with fresh PBS) and analyzed at predetermined time 
intervals by measuring absorbance at $551 \mathrm{~nm}$ using UV spectrophotometer (Cary 60). The concentration/weight of dye released was then calculated from absorbance values, based on the calibration curve obtained for RhB. Finally the release profile was plotted with cumulative release ( $\%$ and weight) against time.

\section{Preparation of bovine trabecular bone cores}

Bone cylinders/cores $(10 \mathrm{~mm}$ diameter and $5 \mathrm{~mm}$ height) were prepared from the sternum harvested from a freshly slaughtered 13-month-old steer, following the procedure described previously. ${ }^{15} 3$ types of bone cores were prepared: with marrow removed/no bone marrow (No $\mathrm{BM})$, with marrow intact $(\mathrm{BM})$, and with marrow + anticoagulant (heparin) $(\mathrm{BM}+\mathrm{HEP})$. For BM + HEP bone cores, heparin was used to prevent coagulation, and was used as an additive throughout the preparation and media perfusion. All bone cores were stored in separate prewash medium [high-glucose Dulbecco's Modified Eagle Medium (Life Technologies Corporation, USA), with $20 \mathrm{mM}$ of 4-(2-hydroxyethyl)-piperazineethanesulfonic acid, $2.4 \mathrm{mg} / \mathrm{mL}$ benzylpenicillin, $3.2 \mathrm{mg} / \mathrm{mL}$ gentamicin sulfate, and $4 \mu \mathrm{g} / \mathrm{mL}$ amphotericin $\mathrm{B}]$ at $4{ }^{\circ} \mathrm{C}$ prior to use for perfusion studies.

\section{Monitoring therapeutic release inside bone cores ex-vivo}

A hole was drilled through the middle of each bone core using a sterilized stainless steel Kirschner wire, and then RhB loaded TNT/Ti wires were carefully inserted into the hole (Figure 2). This was followed by securing the bone into the plastic bone chambers, aligning the implant with the perfusion inlet. Bone culture chambers were then connected to a multi-channel perfusion pump at $37^{\circ} \mathrm{C}$ and culture medium was perfused through the bone samples at the rate 
of $7 \mathrm{ml} / \mathrm{h}$. The perfused media was replaced every three days throughout the experiment. To measure the concentration of the dye released from the TNTs/Ti wires, fluorescence (FL) intensity from the bone cores was measured at pre-determined time intervals, using an in vivo imaging system (Xenogen IVIS 100, Caliper Life Sciences, USA). The imaging mode was set at $5 \mathrm{sec}$ exposure time, medium-size binning, open emission filter and $25 \mathrm{~cm}$ view field, with $2 \times 2$ (width by height) pixel count and a subject area of $0.76 \mathrm{~cm}^{2}$. The captured images were finally processed using Living Image ${ }^{\circledR}$ (version 2.50.1) software to derive FL intensity data representing the concentration of released $\mathrm{RhB}$. Please note that FL intensity only from the bone cores was measured, which directly co-relates with the concentration of dye released into the bone.

\section{Evaluation of integration of TNTs/Ti implants into bone}

At the end of the ex vivo study, bone cores with inserted TNTs/Ti implants were removed from the perfusion chamber and immersed into $20 \mathrm{~mL}$ of fixation solution $(1.4 \% \mathrm{w} / \mathrm{v}$ paraformaldehyde) at room temperature for $24 \mathrm{~h}$ to preserve morphological details. Samples were then placed in a slow decalcifying solution (10\% EDTA, 5\% paraformaldehyde and 50\% PBS, (v/v), $\mathrm{pH} 8$ ) for 3 days before transferring into fast decalcifying media (5\% EDTA, 9.5\% $\mathrm{HNO}_{3},(\mathrm{v} / \mathrm{v})$ ) for $24 \mathrm{~h}$. The samples were placed on photographic film (AGFA Structurix D4 film) and exposed to $35 \mathrm{kV}$ for $2 \frac{1}{2} \mathrm{~min}$ in a Faxitron 804 (Faxitron Company, USA) to confirm decalcification. The soft bone core samples were then carefully cross-sectioned in two ways, (1) with visible implant inserted into bone, and (2) with clear groove caused by circular implant surface. Sample (1) was treated using ethanol to prepare for SEM to observe any morphological damage on the implant surface due to insertion, any organic deposition and bone implant contact. 
Sample (2) was used for histological analysis. The decalcified samples were embedded into paraffin wax, cut into sections of $5 \mu \mathrm{M}$ thickness using a microtome (Leica microsystems, Germany) and collected on silane-coated slides. The sections were dewaxed using histolene, hydrated using ethanol and water, and stained using haematoxylin for nuclear staining before washing in tap water, blueing using 5\% ammonia water and differentiating using $1 \% \mathrm{HCl}$. The slides were again washed, counterstained using eosin, dehydrated using ethanol and cleared using histolene, before finally mounting in permanent mounting media. The prepared slides were then observed for cells adjacent to the implant position or along the edge of the drill hole used for the TNTs/Ti wire insertion using a light microscope.

\section{Statistical analysis}

Student $t$-test (two-tailed) and two-way analysis of variance, followed by Tukey's multiple comparison test, were used to analyze ex vivo drug release data. A value for ${ }^{*} \mathrm{p}<0.05$ and ${ }^{* * *} \mathrm{p}<$ 0.001 were considered significant in each case.

\section{RESULTS}

\section{Characterization of TNTs/Ti wire implants}

To fabricate TNTs on the entire curved surface area of Ti wire with high growth rates and minimal surface defects/cracks, a modified lactic acid electrolyte procedure was used. ${ }^{17}$ The SEM images of the resultant TNTs on Ti wires after anodization at $60 \mathrm{~V}$ for 20 min are presented in Figure 3, and show various features of the nano-engineered implant. Figure 3a indicates uniform coverage of the Ti wire with TNTs, with the presence of small cracks $(0.5$ to $1 \mu \mathrm{m}$ wide), which arise mainly due to volume expansion of the anodic film. High resolution SEM 
images of the cross-section, the top and bottom of the TNTs (Figure 3b-d) reveal well-ordered nanotubes with open pores and closed bottoms, ideal for loading drugs and achieving localized drug release. The average dimensions obtained for TNTs (anodized for $60 \mathrm{~V}$ for $20 \mathrm{~min}$ ) were 17 $\pm 2 \mu \mathrm{m}$ in lengths and $70 \pm 5 \mathrm{~nm}$ in diameters. The growth rate of $850 \mathrm{~nm} / \mathrm{min}$ confirmed a reasonably fast production time of only 20 minutes to make implants of $17-19 \mu \mathrm{m}$ thickness, which can be extended if fabrication of TNTs with greater thickness is required. These TNT dimensions are controllable and can be tuned by varying anodization parameters such as voltage and time. ${ }^{10}$

\section{In-vitro drug release from TNTs/Ti implants}

TGA quantification confirmed a loading of $25.6 \pm 1.5 \mu \mathrm{g} /$ implant (loading capacity of $2.9 \pm 0.8$ $\mu \mathrm{g} / \mathrm{mm}^{2}$ ) of the model hydrophilic drug RhB inside TNTs (with active length on Ti wire $=9$ $\mathrm{mm}$ ). However, as described previously, various drugs/proteins catering to different bone conditions, can be loaded inside the nanotubes and the loading amounts can easily be manipulated by controlling nanotube dimensions. ${ }^{4,18}$ It is noteworthy to mention that drug loading inside the 'vacant' nanotubes is driven by the diffusion of the dye molecules into the nanotubes and also inside the cracks on the anodic film, followed by evaporation of the solvent. This loading mechanism is further enhanced by sonication and vacuum drying, and can easily be controlled by varying dye concentration, immersion/vacuum times, etc.

The release profile of RhB from TNTs in PBS in-vitro is presented in Figure 4, and shows a biphasic release pattern with initial burst release (IBR) for $1^{\text {st }} 6 \mathrm{~h}$, followed by delayed sustained release phase. The release mechanism is diffusion based, dependent on the concentration gradient between the nanotubes (containing $\mathrm{RhB}$ ) and the surrounding PBS. Clearly for the IBR phase, around 37.3 weight $\%$ of the loaded RhB was released in PBS, which 
can be attributed to high concentration gradient when TNTs implants are exposed to releasing buffer, and corresponds to RhB loaded near open pores of the nanotubes and in the cracks on anodic films. Later, $100 \%$ release occurred over 13 days, from the RhB present deep inside the nanotubes. Table 1 summarizes the results from $\mathrm{RhB}$ loading and in-vitro release investigations.

It is also noteworthy that various strategies that have been devised for delaying/controlling the release of loaded drugs from TNTs could also be integrated into our current implant system. ${ }^{18-20}$

\section{Release of model drug from TNTs/Ti implants into trabecular bone ex-vivo}

$\mathrm{RhB}$ loaded implants were inserted into three different bone cores: NoBM, BM and BM+HEP, and were securely placed inside custom bone chambers, prior to connection with perfusion media. With the main aim being monitoring of drug release inside the various bone microenvironments from the surface of nano-engineered implants, FL imaging was performed at predetermined time intervals and the resulting images/intensity plots are presented in Figures 5-6. Likely due to the varied local environments in the vicinity of the implant surface, the dye release inside the bone was different for each bone core studied. This can be attributed to two main factors: the extent of coagulation and effect of perfusion. Briefly, coagulation is expected to block partially or completely the open pores of nanotubes, and also restrict the flow of perfusion media. Also empty and marrow-intact bone cores are expected to pose no restriction and greatly impede the media flow, respectively. As can be seen in Figures 5 and 6, for the NoBM cores, a significant amount of dye is released in the first few hours, after which the release reduces. This can be related to the porous architecture of these cores filled with aqueous media, which allowed rapid initial diffusion of dye (similar to IBR phase with in-vitro release), followed by a period of slow and sustained release observed until the termination of the experiment (day 11). For NoBM, 
the constant perfusion of the cores with culture media would be expected to effectively remove the dye released from TNTs, creating a concentration gradient critical for continued dye release. In the case of bone cores with an intact marrow (BM and BM+HEP), for the initial few hours a low amount of dye was released, which can be attributed to the marrow occupied bone architecture. The presence of heparin appeared to reduce the inhibition of perfusion and hence dye release, likely due to the prevention of blood coagulation. Beyond the first day, for BM and $\mathrm{BM}+\mathrm{HEP}$, a similar pattern continued, with more drug released in presence of heparin. However, after 4-5 days, a similar release pattern was seen in both experimental groups. The large error for each time point for all the bone cores is likely due to the complex and variable internal microarchitectural arrangement of the cores, irrespective of the fact that the samples were isolated from the sternum of a single animal. A summary of the drug release pattern in each experimental condition is presented in Table 2 .

\section{Integration and mechanical stability of TNTs/Ti wire implants}

Bone cores with inserted implants were processed post-experimentally to investigate the effect of bone insertion/removal on the structural integrity of TNTs and to visualize the interaction between implants and the bone. SEM images showing the morphology of the implants within bone are presented in Figures 7-9. For NoBM, the implants showed minimal contact/interaction with the surrounding bone (Figure 7). With the presence of bone marrow (BM and BM+HEP), the implants were observed to form apparently firm attachments with the surrounding bone and marrow (Figures 8-9). Contrary to NoBM, implants in bone marrow also showed some visible deposits, which are likely due to adhesion and deposition of different bone cells, blood cells and marrow contents. To study the impact of implant placement inside the bone cores and media perfusion for extended durations, imaging was also performed to observe the surface of the 
implants. For each type of bone core studied, high magnification images show clear evidence of survival of the nanotube structures. This is potentially important since delamination and release of nanoparticulates may cause local tissue toxicity. ${ }^{21}$ To investigate the effect of implant insertion/placement into the bone, the viability of bone cells adjacent to the implant was observed by histological analysis of decalcified bone. Microscopic observation of these samples (Figure 10) showed the presence of viable osteocytes along the edge of the drilled hole and contact of the implant in all types of bone cores tested after 11 days of the ex vivo study.

\section{DISCUSSION}

The aim of this study was to analyze the release of therapeutics directly inside the bone microenvironment from the surface of nano-engineered bone implants (Figures 1-2). A modified electrochemical anodization procedure using lactic permitted the fabrication of stable anodic film with TNTs on the curved surface of the Ti wire (Figure 3). TNTs have been shown to offer excellent drug elution properties, and have been studied in the context of various bone applications, including infection, osseointegration and cancer. ${ }^{22-24}$ More recently, TNT technology was extended to Ti wires to enable 3D release directly inside the bone matrix. ${ }^{14-16}$ The motivation behind using $\mathrm{Ti}$ wires as an implant substrate is to design minimally invasive therapeutic implants that are able to be inserted into affected bone sites for effective localized therapeutic action. The ability to produce well-ordered and physically robust TNTs on substrates with complex morphologies/geometries such as $\mathrm{Ti}$ wires will enable ready integration into current bone implant types, including pins, meshes and screws.

When anodizing curved surfaces of Ti substrates, cracks predictably occur on the anodic

film, as a result of radial outgrowth of nanotubes and volume expansion of oxide $\left(\mathrm{TiO}_{2}\right)$ on metal 
surfaces. ${ }^{25}$ As we have previously reported, these cracks do not compromise the stability of the anodic film and actually allow for increased loading of therapeutics, albeit subject to burst release kinetics. ${ }^{14-16}$ Using an optimized fabrication procedure to engineer TNTs with fewer surface cracks, the amount and release kinetics of a particular drug could be tailored to achieve a an optimal release profile, as presented in Figure 4. For example, too high an initial burst release can cause local tissue toxicity. ${ }^{3-4}$ To further control drug release, a number of emerging technologies for TNTs, including multi-drug loading, and controlled/triggered release, could also be integrated into the current TNTs/Ti wire system. ${ }^{18}$ The TNTs/Ti wire implant system thus represents an accessible and scalable technology with modifiable loading and release, suitable for local drug delivery in a number of bone conditions.

While many strategies have been proposed for delivering therapeutics directly at the target bone site from the surface of the implants, the therapeutic distribution or release inside the bone micro-environment has not been explored. Most of the studies proposing therapeutic functions of TNTs modified Ti implants include in-vitro or in-vivo studies. Here, proof of principle was therefore sought using a bioreactor capable of culturing large animal-derived trabecular bone for extended periods ex vivo. ${ }^{26-28} \mathrm{RhB}$ loaded implants were inserted inside bovine bone cores, with varied micro-environments. It was found that for cores containing bone marrow, a very low amount of drug was released initially, with continual release thereafter. The SEM images of the implant retrieved from the BM bone core (Figure 8c) also show attachment of marrow and cells on the surface of the implant, which is expected to block a proportion of pores on the implant.

In contrast, for NoBM samples, there was a quick release of drug payloads initially, followed by a slow and sustained release. Thus the NoBM system was similar to in-vitro release 
(Figure 4), in which a biphasic release pattern was observed. Effective perfusion of the trabecular bone matrix resulted in the continuous clearance of the media, maintaining a concentration gradient around the implant surface, which contributed to the continued drug release. We have shown previously that in the absence of culture flow, the spread of therapeutics inside the bone matrix (without marrow) is very slow. ${ }^{15}$ In the absence of marrow, a reduced amount of deposition was seen on the implants, again allowing free release of drug. The third type of bone core investigated was trabecular bone with marrow treated with heparin (BM+HEP), which was investigated to determine the effect of inhibiting blood clotting on drug release from the implants. With the addition of heparin, it was found that the release of drug payloads inside the bone core was initially intermediate between the BM and the NoBM conditions, and thereafter similar. Examination of the implant after retrieval from BM+HEP (Figure 9c) indicated significant blockage of pores attributed to anchoring/attachment of cells from the bone marrow onto the implant surface.

The surface morphology of the nano-engineered implants after termination of the ex-vivo drug diffusion study (Figures 7-9) confirmed the fabrication of stable and robust TNTs onto the complex substrate of $\mathrm{Ti}$ wire. The surface texture appeared unchanged after insertion and removal from the bone, which addresses a major concern with this approach, namely that of the delamination of anodic film during physical handling. ${ }^{6,21}$ In addition, the presence of viable bone cells adjacent to implant surface (Figure 10) indicates that neither the TNTs surface nor the needle puncture approach used for implant placement affected the health of the adjacent bone tissue. This observation is consistent with other reports of the biocompatibility of TNTs. ${ }^{12,13,23}$ To summarize, the results shown in this ex vivo model are consistent with the ability of the nano-engineered Ti wire implants to address complex therapeutic challenges of severe bone 
traumas. The optimized fabrication procedure enabled growth of stable anodic films on Ti wires, with the ability to load substantial amounts of drugs, and achieve local elution directly inside the bone matrix. Analysis of the drug distribution inside the bone microenvironment using $\operatorname{ZetOS}^{\mathrm{TM}}$ system, enabled an understanding of varied release profiles dependent on the presence of bone marrow, the ability to maintain a diffusion gradient and continued media perfusion. This system clearly has good potential to test and optimize drug releasing implants for specific bone conditions.

\section{CONCLUSION}

In the current study, nano-engineered Ti wires with TNTs were proposed as therapeutic bone implants, and their ability to release loaded drugs locally in a 3D fashion directly inside the bone micro-environment using an ex-vivo bone model was demonstrated. Using an optimized anodization procedure, the fabrication of well-adherent TNTs on the entire surface area of Ti wire was obtained. Implants were loaded with a model hydrophilic drug, $\mathrm{RhB}$, and placed inside bovine trabecular bone cores ex-vivo using a needle puncture approach. In-situ fluorescence intensity measurements established varied release kinetics/spread of the model drug inside the bone cores, with slower release and high drug retention observed for marrow occupied bone cores. Imaging the implants after retrieval from the bone tissue established bone-implant contact and survival of the nanotubes through the process. We propose that such an approach could be used to target local skeletal conditions such as infection and cancer.

\section{ACKNOWLEDGMENTS}

The authors acknowledge the financial support of the Australian Research Council (FT110100711 and DP 120101680) and the University of Adelaide for this work. Authors also 
acknowledge the technical support from Dr Agatha Labrinidis at The Adelaide Microscopy, The University of Adelaide.

\section{REFERENCES}

1. Buchholz H, Elson R, Engelbrecht E, Lodenkamper H, Rottger J, Siegel A. Management of deep infection of total hip replacement. Bone Joint J 1981;63-B:342-353.

2. Popat KC, Eltgroth M, Latempa TJ, Grimes CA, Desai TA. Decreased staphylococcus epidermis adhesion and increased osteoblast functionality on antibiotic-loaded titania nanotubes. Biomaterials 2007;28:4880-4888.

3. Gulati K, Aw MS, Findlay D, Losic D. Local drug delivery to the bone by drug-releasing implants: Perspectives of nano-engineered titania nanotube arrays. Ther Deliv 2012;3:857-873.

4. Losic D, Aw MS, Santos A, Gulati K, Bariana M. Titania nanotube arrays for local drug delivery: Recent advances and perspectives. Expert Opin Drug Deliv 2015;12:103-127.

5. Bauer S, Schmuki P, Von Der Mark K, Park J. Engineering biocompatible implant surfaces: Part I: Materials and surfaces. Prog Mater Sci 2013;58:261-326.

6. Santos A, Aw MS, Bariana M, Kumeria T, Wang Y, Losic D. Drug-releasing implants: Current progress, challenges and perspectives. J Mater Chem B 2014;2:6157-6182.

7. Kieswetter K, Schwartz Z, Hummert TW, Cochran DL, Simpson J, Dean DD, Boyan BD. Surface roughness modulates the local production of growth factors and cytokines by osteoblastlike MG-63 cells. J Biomed Mater Res 1996;32:55-63.

8. Wong M, Eulenberger J, Schenk R, Hunziker E. Effect of surface topology on the osseointegration of implant materials in trabecular bone. J Biomed Mater Res 1995;29:15671575. 
9. Yao C, Slamovich EB, Webster TJ. Enhanced osteoblast functions on anodized titanium with nanotube-like structures. J Biomed Mater Res A 2008;85A:157-166.

10. Roy P, Berger S, Schmuki P. TiO 2 nanotubes: Synthesis and applications. Angew Chem Int Ed 2011;50:2904-2939.

11. Yao C, Webster TJ. Prolonged antibiotic delivery from anodized nanotubular titanium using a co-precipitation drug loading method. J Biomed Mater Res B Appl Biomater 2009;91B:587-595.

12. Von Wilmowsky C, Bauer S, Lutz R, Meisel M, Neukam FW, Toyoshima T, Schmuki P, Nkenke E, Schlegel KA. In vivo evaluation of anodic $\mathrm{TiO}_{2}$ nanotubes: An experimental study in the pig. J Biomed Mater Res B Appl Biomater 2009;89B:165-171.

13. Popat KC, Leoni L, Grimes CA, Desai TA. Influence of engineered titania nanotubular surfaces on bone cells. Biomaterials 2007;28:3188-3197.

14. Gulati K, Aw MS, Losic D. Drug-eluting Ti wires with titania nanotube arrays for bone fixation and reduced bone infection. Nanoscale Res Lett 2011;6:571.

15. Aw MS, Khalid KA, Gulati K, Atkins GJ, Pivonka P, Findlay DM, Losic D. Characterization of drug-release kinetics in trabecular bone from titania nanotube implants. Int $\mathbf{J}$ Nanomedicine $2012 ; 7: 4883-4892$.

16. Gulati K, Atkins GJ, Findlay DM, Losic D. Nano-engineered titanium for enhanced bone therapy. Proc SPIE 8812, Biosensing and Nanomedicine VI, 88120C (September 11, 2013); doi:10.1117/12.2027151.

17. So S, Lee K, Schmuki P. Ultrafast growth of highly ordered anodic TiO2 nanotubes in lactic acid electrolytes. J Am Chem Soc 2012;134:11316-11318. 
18. Aw MS, Kurian M, Losic D. Non-eroding drug-releasing implants with ordered nanoporous and nanotubular structures: Concepts for controlling drug release. Biomater Sci 2014;2:10-34.

19. Gulati K, Ramakrishnan S, Aw MS, Atkins GJ, Findlay DM, Losic D. Biocompatible polymer coating of titania nanotube arrays for improved drug elution and osteoblast adhesion. Acta Biomater 2012;8:449-456.

20. Aw M, Gulati K, Losic D. Controlling drug release from titania nanotube arrays using polymer nanocarriers and biopolymer coating. Biomater Nanobiotechnol 2011;2:477-484.

21. Geetha M, Singh AK, Asokamani R, Gogia AK. Ti based biomaterials, the ultimate choice for orthopaedic implants -A review. Prog Mater Sci 2009;54:397-425.

22. Ma M, Kazemzadeh-Narbat M, Hui Y, Lu S, Ding C, Chen DDY, Hancock REW, Wang R. Local delivery of antimicrobial peptides using self-organized $\mathrm{TiO}_{2}$ nanotube arrays for periimplant infections. J Biomed Mater Res A 2012;100A:278-285.

23. Bjursten LM, Rasmusson L, Oh S, Smith GC, Brammer KS, Jin S. Titanium dioxide nanotubes enhance bone bonding in vivo. J Biomed Mater Res A 2010;92A:1218-1224.

24. Kalbacova M, Macak JM, Schmidt-Stein F, Mierke CT, Schmuki P. TiO2 nanotubes: Photocatalyst for cancer cell killing. Phys Status Solidi Rapid Res Lett 2008;2:194-196.

25. Gulati K, Santos A, Findlay D, Losic D. Optimizing anodization conditions for fabricating welladherent and robust titania nanotubes on curved surfaces. J Phy Chem C $2015 ; 119: 16033-16045$.

26. Schnieders J, Gbureck U, Germershaus O, Kratz M, Jones DB, Kissel T. Ex Vivo Human Trabecular Bone Model for Biocompatibility Evaluation of Calcium Phosphate Composites 
Modified with Spray Dried Biodegradable Microspheres. Adv Healthcare Mater 2013;2:13611369.

27. Jones DB, Broeckmann E, Pohl T, Smith EL. Development of a mechanical testing and loading system for trabecular bone studies for long term culture. Eur Cell Mater 2003;5:48-59.

28. Endres S, Kratz M, Wunsch S, Jones DB. Zetos: a culture loading system for trabecular bone. Investigation of different loading signal intensities on bovine bone cylinders. J Musculoskelet Neuronal Interact 2009;9:173-183.

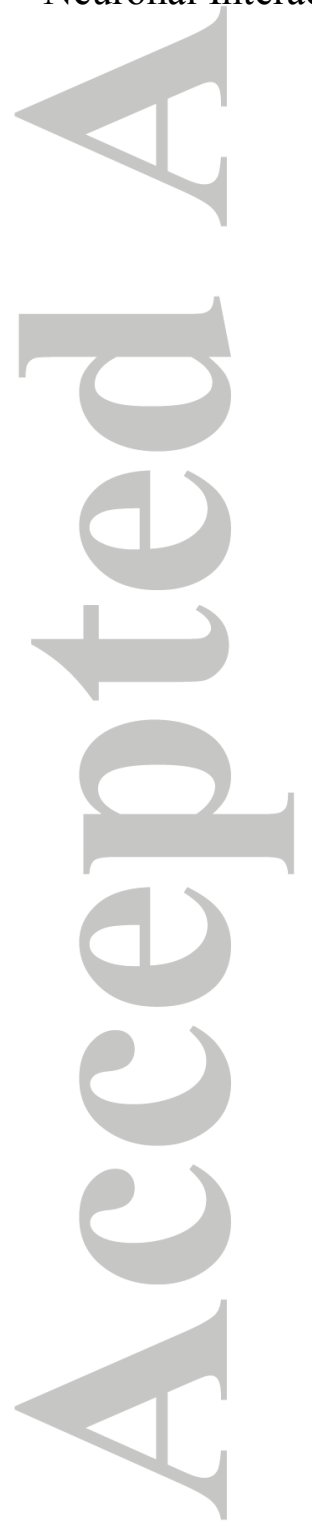

John Wiley \& Sons, Inc. 


\section{FIGURES CAPTIONS}

FIGURE 1. Scheme showing TNTs/Ti wire implants proposed for in-bone therapeutic application. (a) Fabrication of TNTs on Ti wire, (b) loading drugs inside the TNTs, and (c) implantation of drug loaded TNTs inside the traumatized bone for local drug delivery. [TNTs: titania nanotubes]

FIGURE 2. Monitoring the release of therapeutics inside the trabecular bone ex-vivo from the surface of TNTs/Ti wire implants: (a) harvesting sternum from bovine animal, preparation of bone cylinders/cores and drilling a hole using stainless steel Kirschner wire, (b) placement of RhB loaded TNTs/Ti wire implants inside the hole, (c) securing the bone core inside the perfusion chamber with wire orientation parallel to inlet, (d) attaching the bone chamber to a media perfusion pump, and (e) monitoring the release of $\mathrm{RhB}$ inside the bone core using fluorescence imaging.

FIGURE 3. Structural morphology of TNTs/Ti wire implants. SEM images of (a) prepared TNTs/Ti wire implants [inset: digital image of the whole implant], (b) a cross-sectional view showing TNT length or thickness, (c) the top surface showing open pores of TNTs, and (d) bottom surface showing closed end of the nanotubes (image taken by removing TNT layer from Ti wire).

FIGURE 4. In vitro release of $\mathrm{RhB}$ from $\mathrm{TNTs} / \mathrm{Ti}$ wire implants into $\mathrm{PBS}(\mathrm{pH}$ 7.4). Graph indicating (a) cumulative percentage (\%) and amount released over 13 days, and (b) first $6 \mathrm{~h}$ release (burst release). Data represent means $\pm \mathrm{SD}, \mathrm{n}=3$. 


\section{(c)}

FIGURE 5. Top-view fluorescence images of the bone cores (inner circle, secured in bone chambers: outer circle) representing change in intensity corresponding to dye diffusion from the surface of TNTs/Ti wire implants.

(2)

FIGURE 6. Release profile of RhB from TNTs/Ti wire implants inside the trabecular bone cores ex-vivo: (a) changes in FL intensities (corresponding to $\mathrm{RhB}$ concentration), and (b) release pattern observed in first $6 \mathrm{~h}$, with an inset showing graph expansion.

FIGURE 7. Stability and interaction of TNTs/Ti wire implants inside marrow-less bone (NoBM). (a) Cross-sectional digital photo and SEM images: (b-c) implant inside the bone, and (d) top-view of the implant.

FIGURE 8. Stability and interaction of TNTs/Ti wire implants inside marrow-containing bone (BM). (a) Cross-sectional digital photo and SEM images: (b-c) implant inside the bone, and (d) top-view of the implant. Arrows indicate adhesion/attachment between the bone and the implant surface.

FIGURE 9. Stability and interaction of TNTs/Ti wire implants inside heparin-treated marrowcontaining bone (BM+HEP). (a) Cross-sectional digital photo and SEM images: (b-c) implant inside the bone, and (d) top-view of the implant. Arrows indicate adhesion/attachment between the bone and the implant surface. 


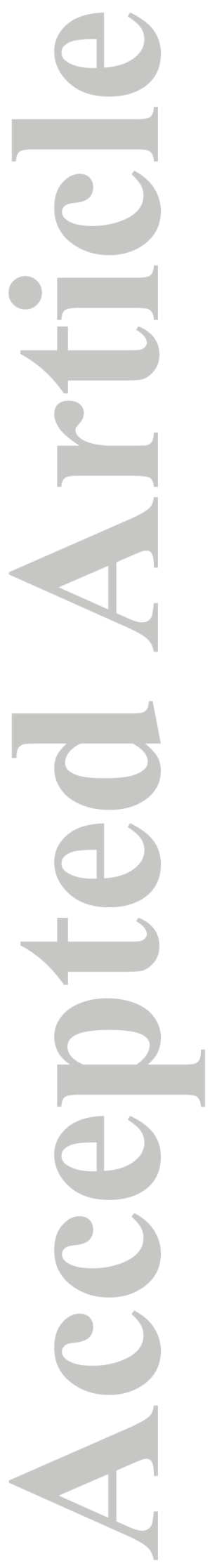

John Wiley \& Sons, Inc. 
FIGURE 10. Histology of bone cores inserted with TNTs/Ti wire implants after termination of the perfusion/drug release study ex-vivo. (a) Digital image indicating a representative hematoxylin-eosin stained histology sample (arrow indicating implant position). Light microscopy images showing presence of viable osteocytes (blue stained nuclei in white lacunae) along the drilled areas in the bone cores: (b) no bone marrow (NoBM), (c) presence of marrow (BM), and (d) heparin treated bone marrow (BM+HEP).
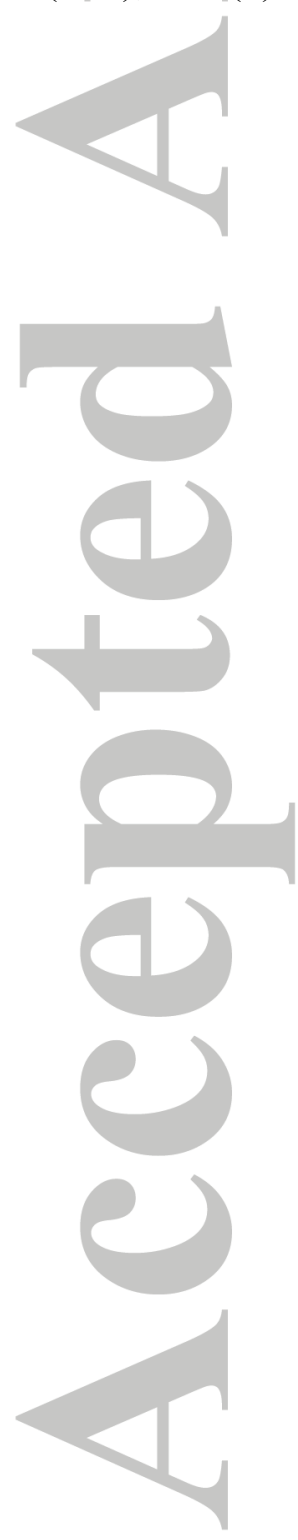

John Wiley \& Sons, Inc. 


\section{TABLES CAPTIONS}

TABLE 1. Summary of RhB loading and in vitro release from TNTs/Ti wire implants.

TABLE 2. Factors influencing release of model drug $(\mathrm{RhB})$ from the surface of TNTs/Ti wire implants directly inside the bone microenvironment ex-vivo.
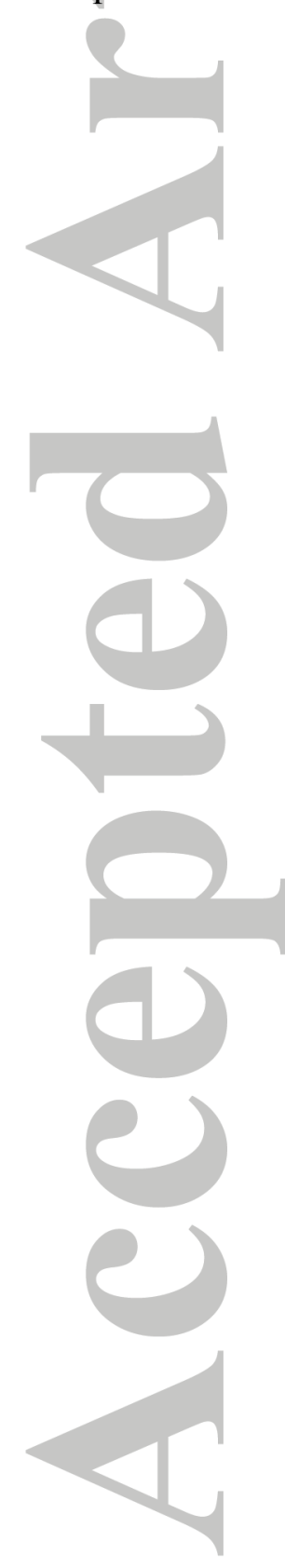

John Wiley \& Sons, Inc. 


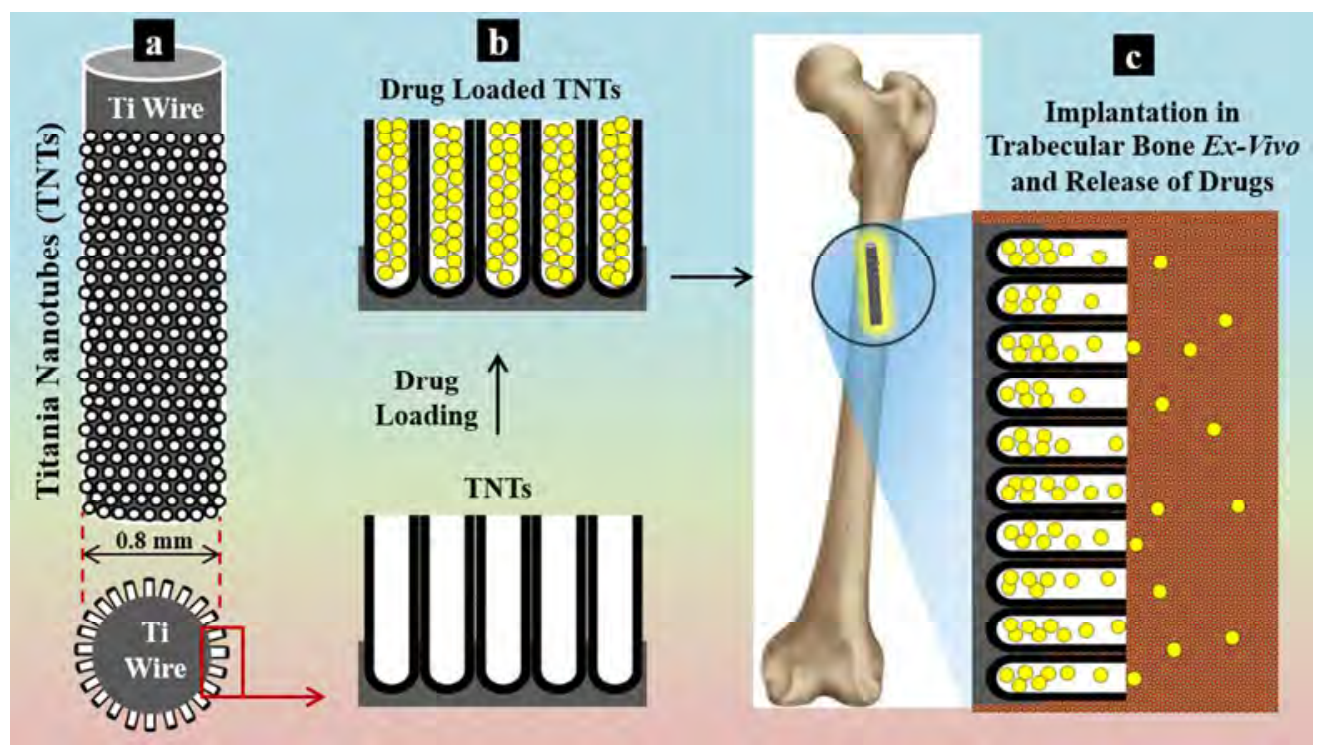

FIGURE 1. Scheme showing TNTs/Ti wire implants proposed for in-bone therapeutic application. (a) Fabrication of TNTs on Ti wire, (b) loading drugs inside the TNTs, and (c) implantation of drug loaded TNTs inside the traumatized bone for local drug delivery. [TNTs: titania nanotubes]

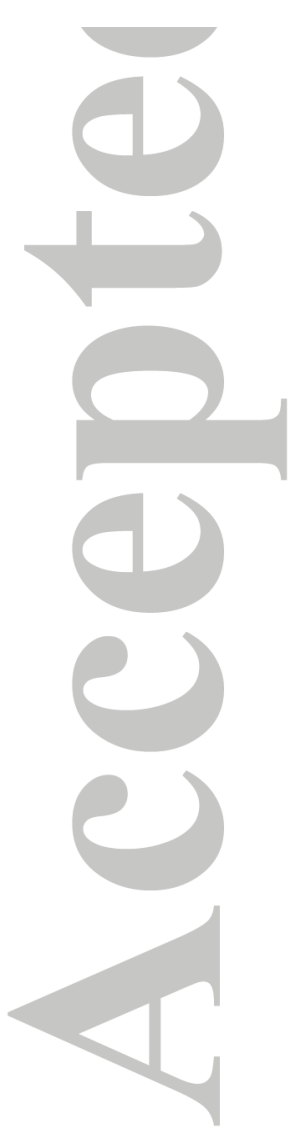
$94 \times 53 \mathrm{~mm}(300 \times 300 \mathrm{DPI})$ 

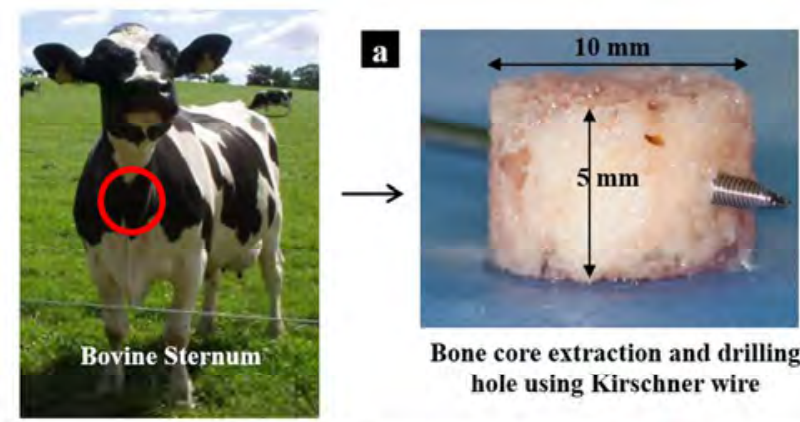

Bone core extraction and drilling hole using Kirschner wire

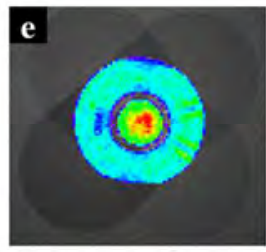

Fluorescence Imaging (top-view of bone core)

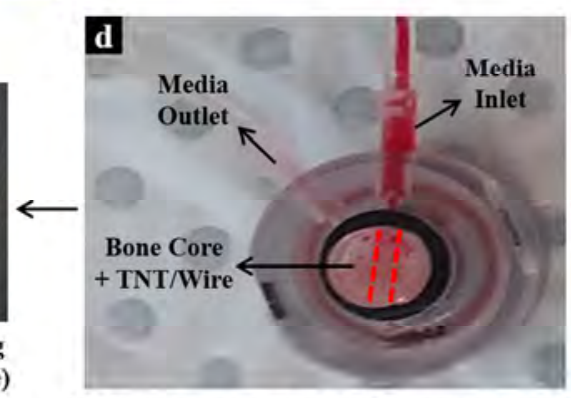

Media Flow using ZetOS ${ }^{\mathrm{TM}}$ System (top-view)

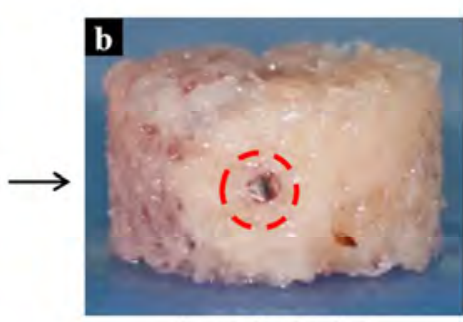

Insert dye loaded TNT/Ti Wire

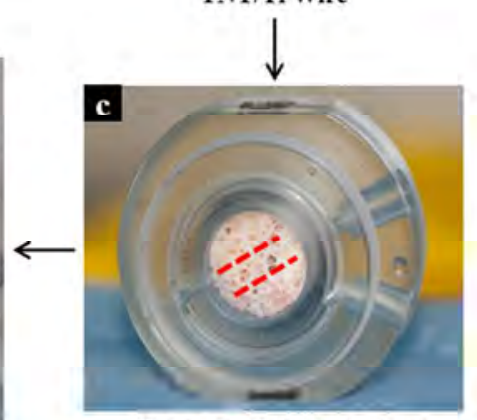

Bone secured inside media perfusion chamber (bottom-view)

FIGURE 2. Monitoring the release of therapeutics inside the trabecular bone ex-vivo from the surface of TNTs/Ti wire implants: (a) harvesting sternum from bovine animal, preparation of bone cylinders/cores and drilling a hole using stainless steel Kirschner wire, (b) placement of RhB loaded TNTs/Ti wire implants inside the hole, (c) securing the bone core inside the perfusion chamber with wire orientation parallel to inlet, (d) attaching the bone chamber to a media perfusion pump, and (e) monitoring the release of RhB inside the bone core using fluorescence imaging. $104 \times 70 \mathrm{~mm}(300 \times 300$ DPI $)$ 


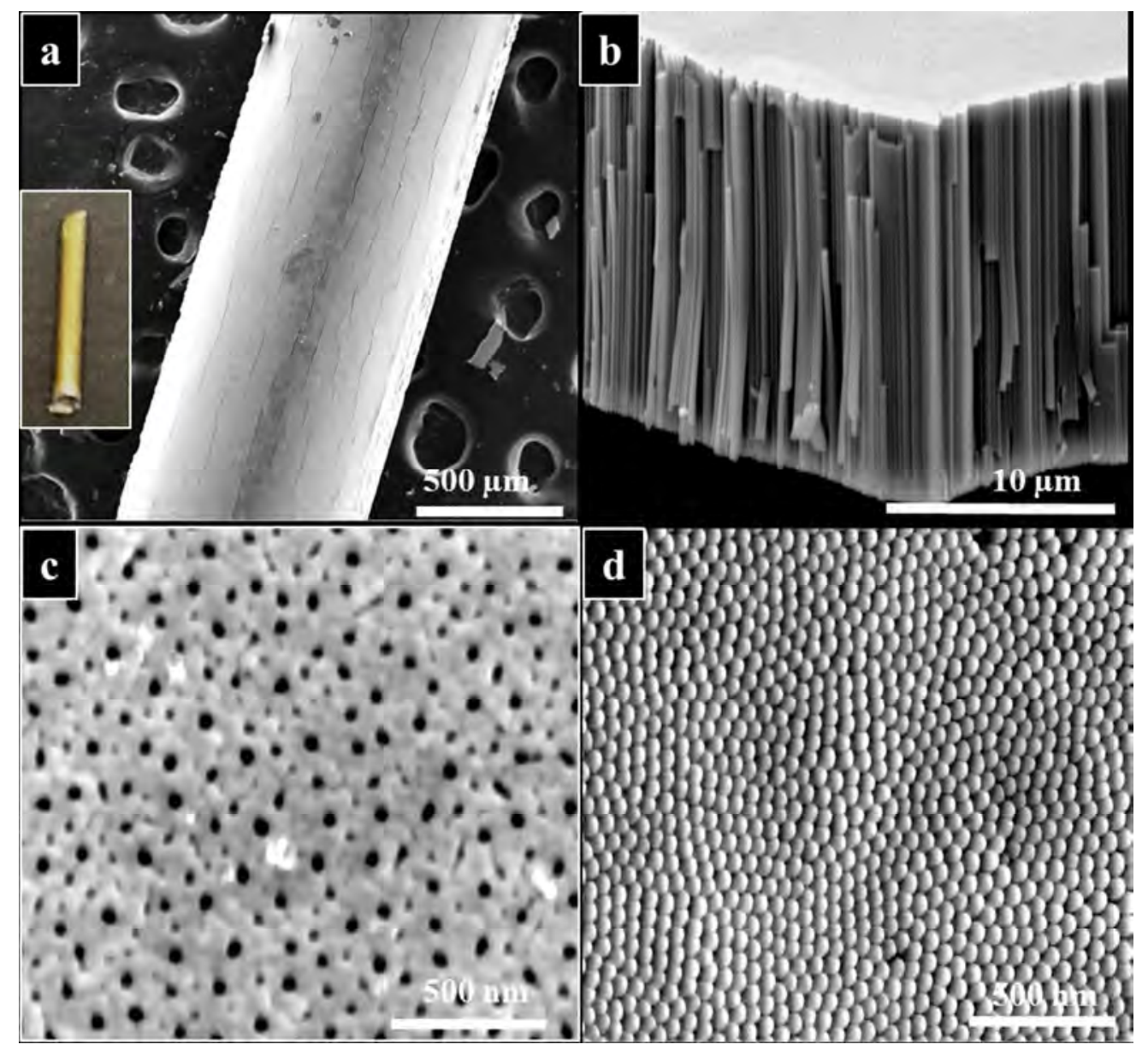

FIGURE 3. Structural morphology of TNTs/Ti wire implants. SEM images of (a) prepared TNTs/Ti wire implants [inset: digital image of the whole implant], (b) a cross-sectional view showing TNT length or thickness, (c) the top surface showing open pores of TNTs, and (d) bottom surface showing closed end of the nanotubes (image taken by removing TNT layer from Ti wire). $80 \times 75 \mathrm{~mm}(300 \times 300 \mathrm{DPI})$ 


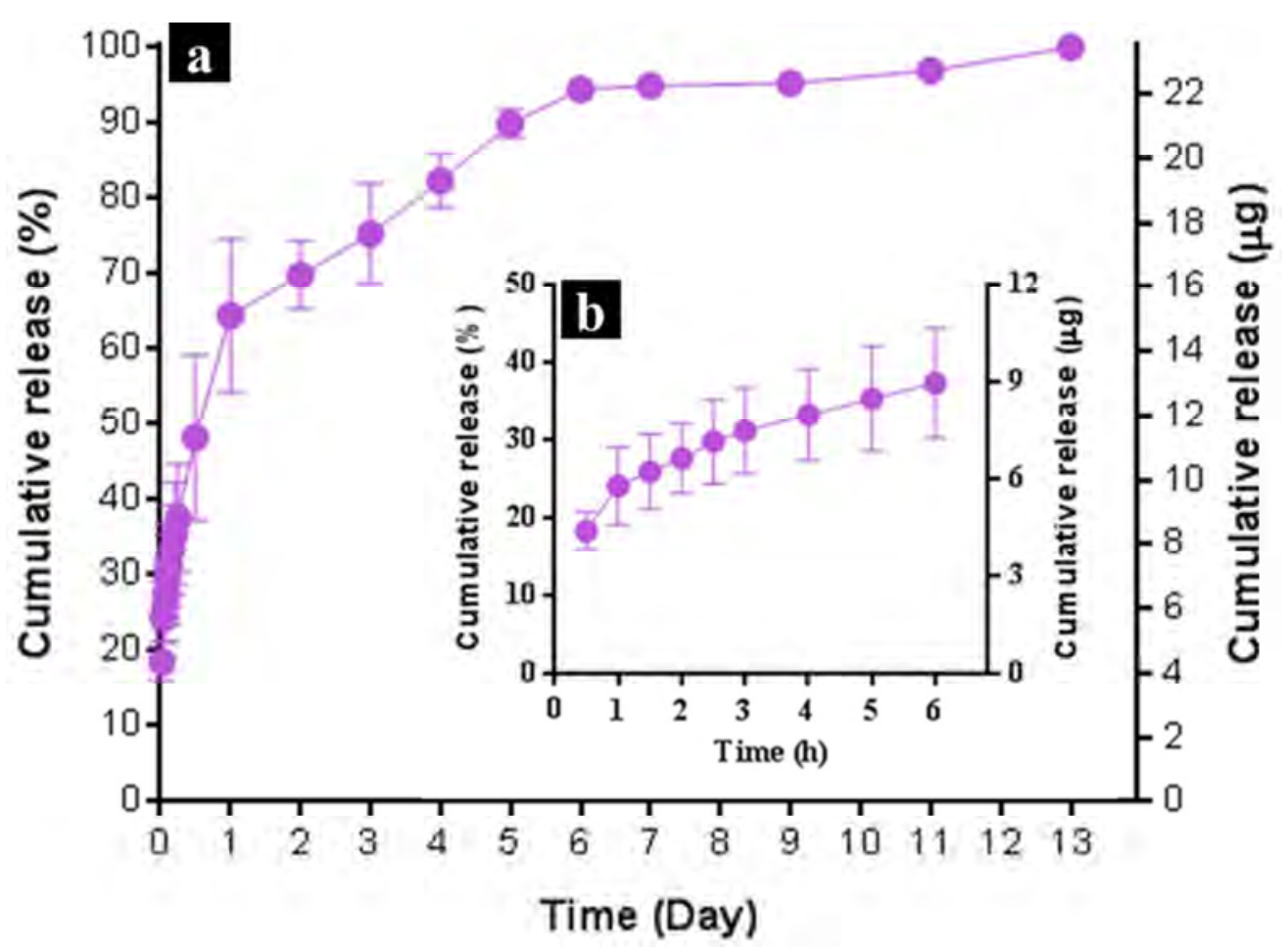

FIGURE 4. In vitro release of RhB from TNTs/Ti wire implants into PBS (pH 7.4). Graph indicating (a) cumulative percentage (\%) and amount released over 13 days, and (b) first $6 \mathrm{~h}$ release (burst release). Data represent means $\pm S D, n=3$. $63 \times 45 \mathrm{~mm}(300 \times 300 \mathrm{DPI})$

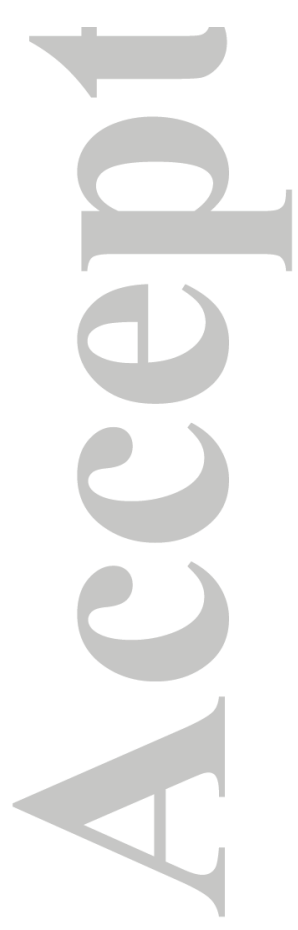

John Wiley \& Sons, Inc.

This article is protected by copyright. All rights reserved. 


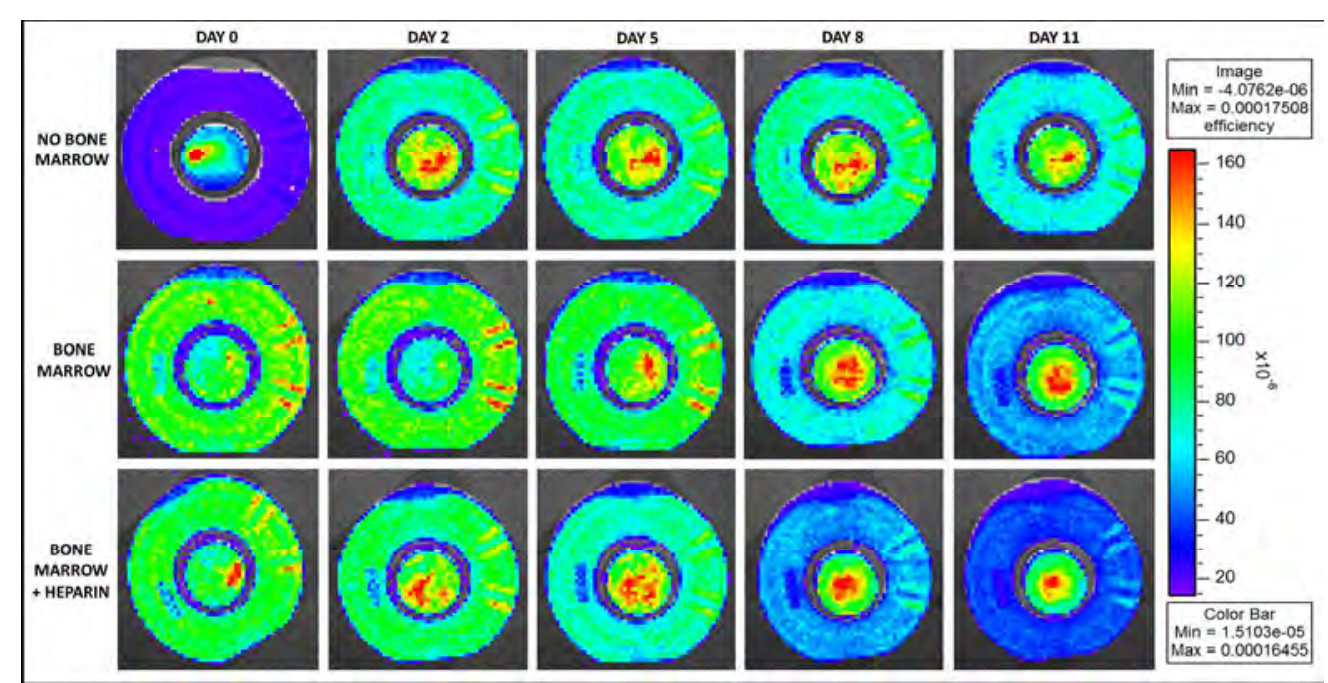

FIGURE 5. Top-view fluorescence images of the bone cores (inner circle, secured in bone chambers: outer circle) representing change in intensity corresponding to dye diffusion from the surface of TNTs/Ti wire implants.

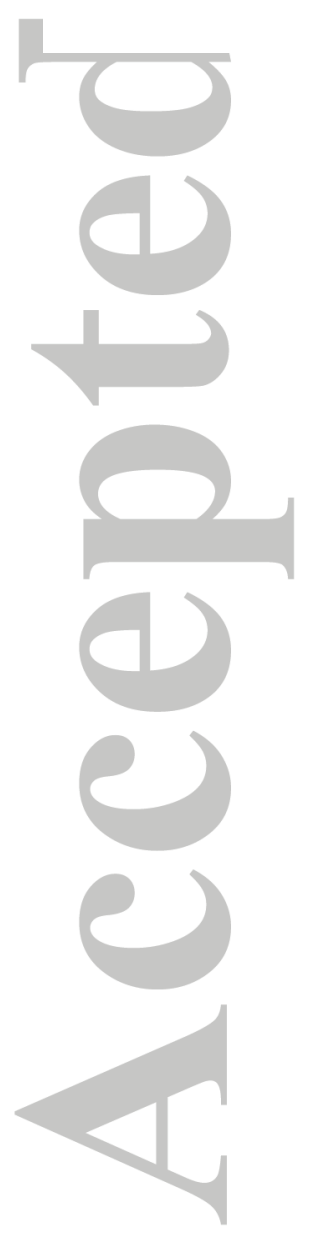

$260 \times 132 \mathrm{~mm}(300 \times 300$ DPI $)$

John Wiley \& Sons, Inc.

This article is protected by copyright. All rights reserved. 

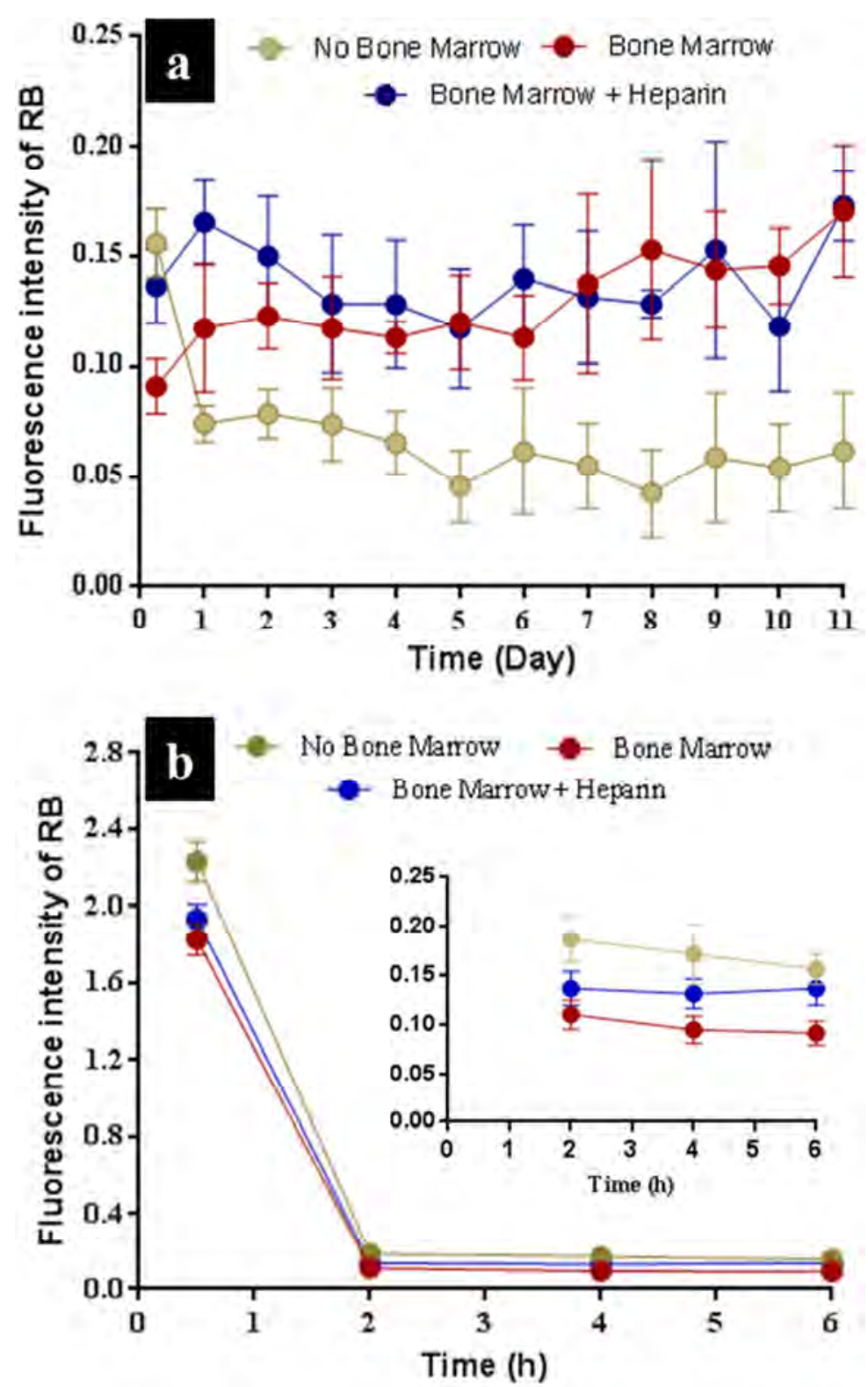

FIGURE 6. Release profile of RhB from TNTs/Ti wire implants inside the trabecular bone cores ex-vivo: (a) changes in FL intensities (corresponding to RhB concentration), and (b) release pattern observed in first 6 , with an inset showing graph expansion.

$51 \times 83 \mathrm{~mm}(300 \times 300 \mathrm{DPI})$

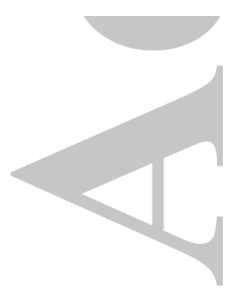

John Wiley \& Sons, Inc.

This article is protected by copyright. All rights reserved. 


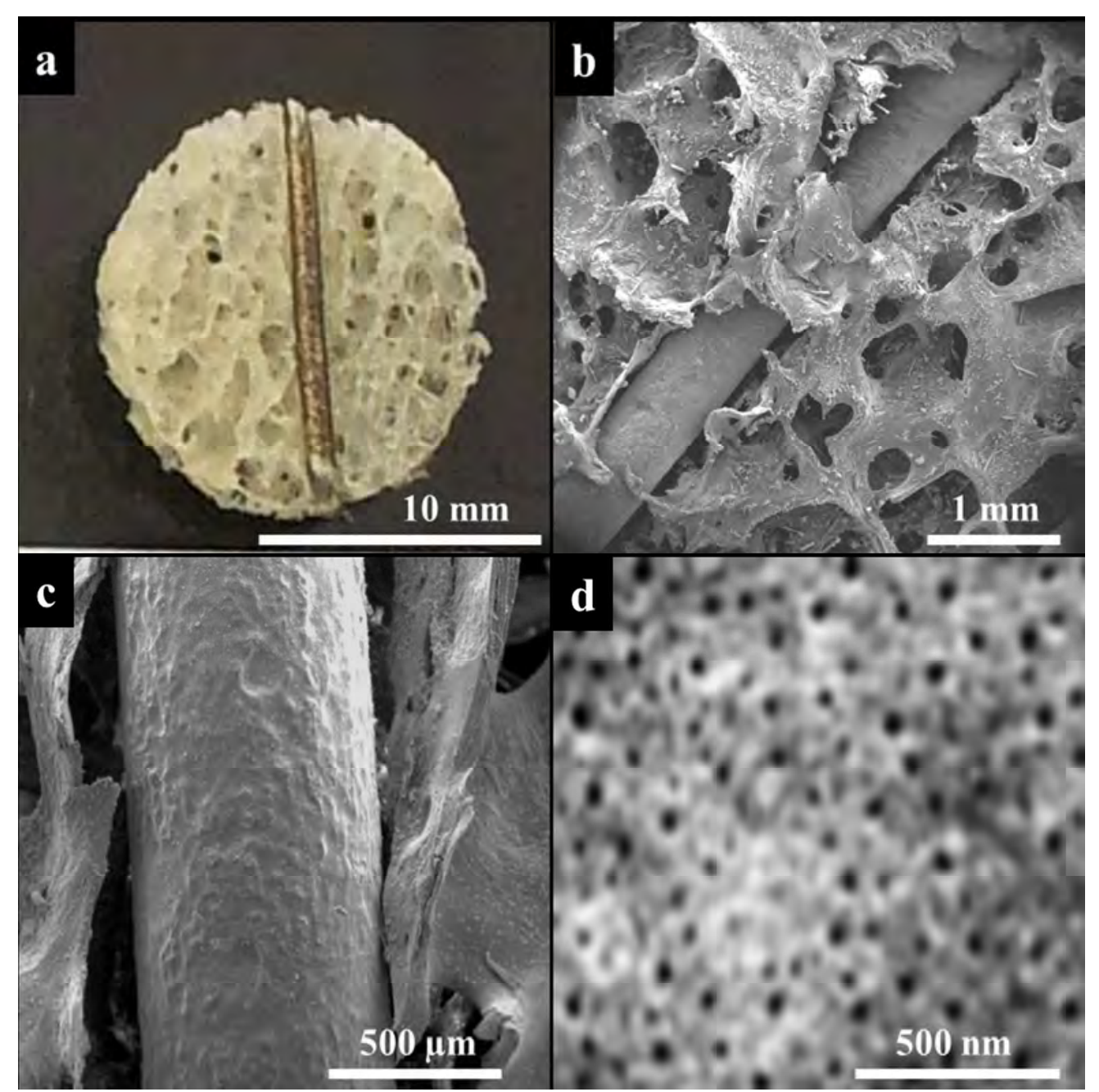

FIGURE 7. Stability and interaction of TNTs/Ti wire implants inside marrow-less bone (NoBM). (a) Crosssectional digital photo and SEM images: (b-c) implant inside the bone, and (d) top-view of the implant.

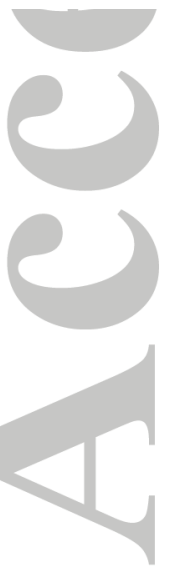
$80 \times 80 \mathrm{~mm}(300 \times 300$ DPI $)$

John Wiley \& Sons, Inc. 


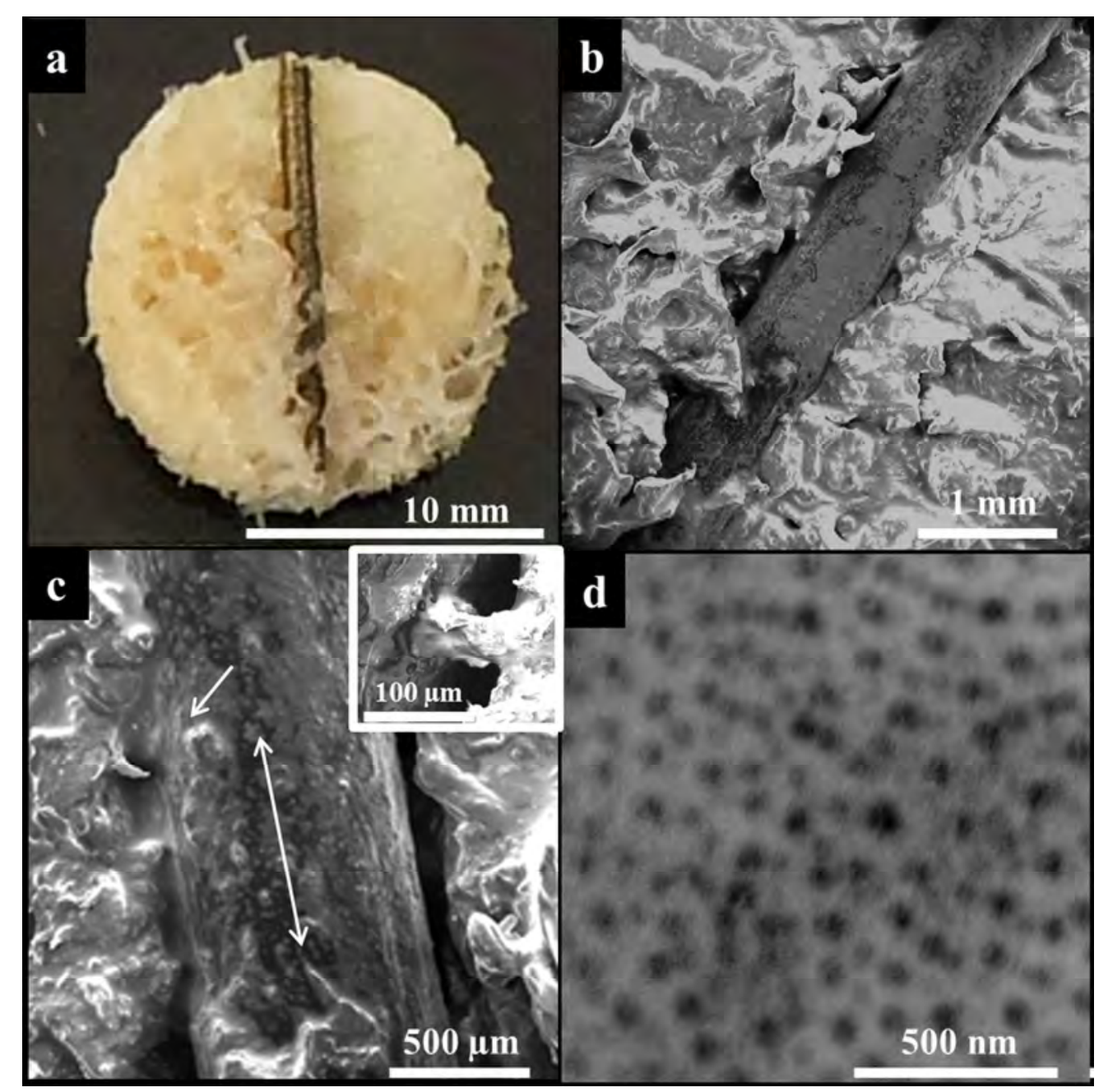

FIGURE 8. Stability and interaction of TNTs/Ti wire implants inside marrow-containing bone (BM). (a) Crosssectional digital photo and SEM images: (b-c) implant inside the bone, and (d) top-view of the implant. Arrows indicate adhesion/attachment between the bone and the implant surface. $81 \times 80 \mathrm{~mm}(300 \times 300 \mathrm{DPI})$

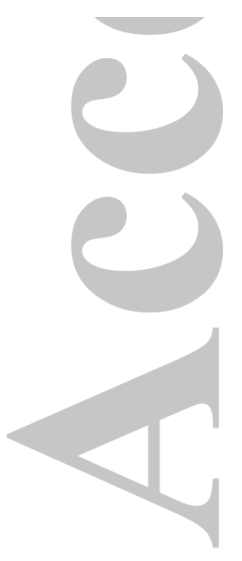

John Wiley \& Sons, Inc. 


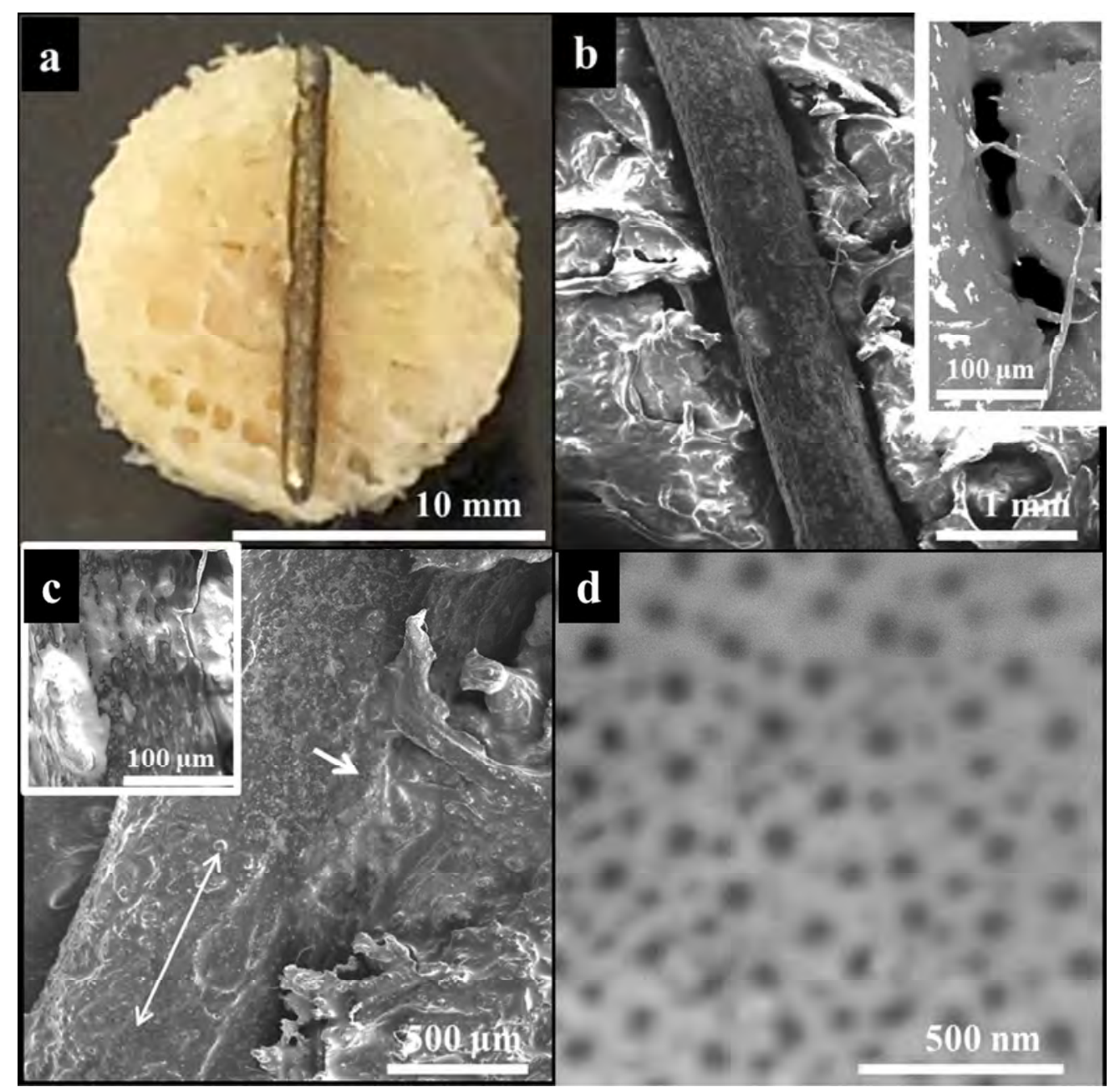

FIGURE 9. Stability and interaction of TNTs/Ti wire implants inside heparin-treated marrow-containing bone $(B M+H E P)$. (a) Cross-sectional digital photo and SEM images: (b-c) implant inside the bone, and (d) topview of the implant. Arrows indicate adhesion/attachment between the bone and the implant surface. $81 \times 80 \mathrm{~mm}(300 \times 300 \mathrm{DPI})$

John Wiley \& Sons, Inc.

This article is protected by copyright. All rights reserved. 


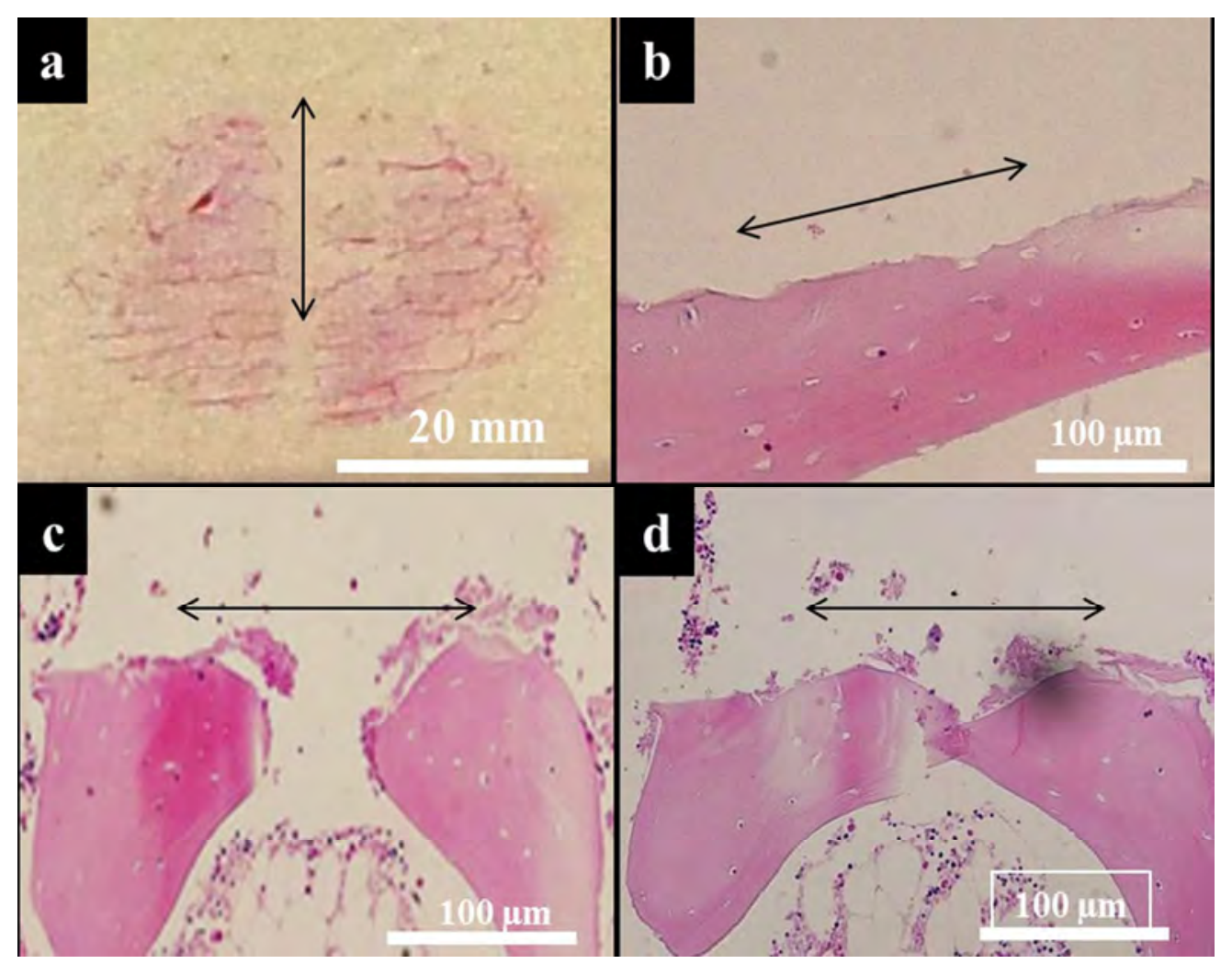

FIGURE 10. Histology of bone cores inserted with TNTs/Ti wire implants after termination of the perfusion/drug release study ex-vivo. (a) Digital image indicating a representative hematoxylin-eosin " stained histology sample (arrow indicating implant position). Light microscopy images showing presence of viable osteocytes (blue stained nuclei in white lacunae) along the drilled areas in the bone cores: (b) no bone marrow (NoBM), (c) presence of marrow (BM), and (d) heparin treated bone marrow (BM+HEP).

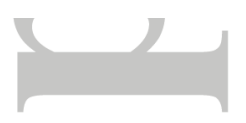
$70 \times 55 \mathrm{~mm}(300 \times 300$ DPI $)$ 


\section{TABLES}

\begin{tabular}{|c|c|c|c|c|c|}
\hline $\begin{array}{c}\text { Nanotube } \\
\text { length }(\mu \mathrm{m})\end{array}$ & $\begin{array}{c}\text { Nanotube } \\
\text { diameter }(\mathrm{nm})\end{array}$ & $\begin{array}{c}\text { Amount of } \\
\text { drug loaded } \\
(\mu \mathrm{g})\end{array}$ & $\begin{array}{c}\text { Drug loading } \\
\text { capacity } \\
\left(\mu \mathrm{g} / \mathrm{mm}^{2}\right)\end{array}$ & $\begin{array}{c}\text { Cumulative } \\
\text { burst release } \\
\text { in } 6 \mathrm{~h}(\%)\end{array}$ & $\begin{array}{c}\text { Time for } \\
100 \% \text { drug } \\
\text { release (days) }\end{array}$ \\
\hline $17 \pm 2$ & $70 \pm 5$ & $25.6 \pm 1.5$ & $2.9 \pm 0.8$ & $37.3 \pm 4.3$ & 13 \\
\hline
\end{tabular}

TABLE 1. Summary of RhB loading and in vitro release from TNTs/Ti wire implants.

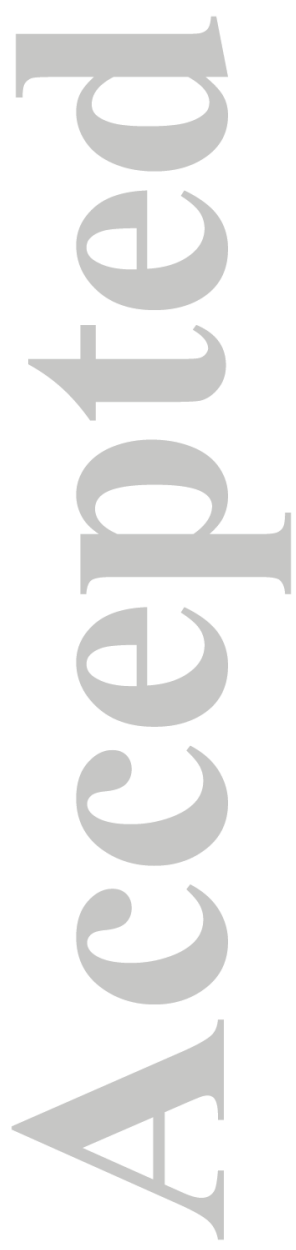

John Wiley \& Sons, Inc.

This article is protected by copyright. All rights reserved. 


\begin{tabular}{|c|c|c|c|}
\hline $\begin{array}{c}\text { Factors } \\
\text { Influencing Drug } \\
\text { Diffusion }\end{array}$ & $\begin{array}{c}\text { No Bone Marrow } \\
\text { (No BM) }\end{array}$ & $\begin{array}{c}\text { Bone Marrow } \\
\text { (BM) }\end{array}$ & $\begin{array}{c}\text { Bone Marrow + } \\
\text { Heparin } \\
(\text { BM + HEP })\end{array}$ \\
\hline ation & N.A. & $\begin{array}{l}\text { Marrow coagulates and } \\
\text { impedes media } \\
\text { perfusion and drug } \\
\text { diffusion }\end{array}$ & $\begin{array}{l}\text { Marrow coagulation is } \\
\text { prevented via addition } \\
\text { of anti-coagulant (HEP) }\end{array}$ \\
\hline $\begin{array}{l}\text { Diffusion of drug } \\
\text { from TNTs inside } \\
\text { the bone core }\end{array}$ & $\begin{array}{c}\text { Very fast, as empty } \\
\text { porous architecture of } \\
\text { bone (filled with media) } \\
\text { increases the diffusion } \\
\text { gradient }\end{array}$ & $\begin{array}{l}\text { Presence of coagulated } \\
\text { marrow blocks the open } \\
\text { pores of TNTs and } \\
\text { results in very low } \\
\text { release }\end{array}$ & $\begin{array}{l}\text { Presence of marrow } \\
\text { (which is uncoagulated) } \\
\text { partially impedes the } \\
\text { diffusion of dye }\end{array}$ \\
\hline $\begin{array}{l}\text { Perfusion of } \\
\text { media in the bone } \\
\text { core }\end{array}$ & $\begin{array}{l}\text { Very effective and } \\
\text { results in quick removal } \\
\text { of drug molecules from } \\
\text { inside the bone, thereby } \\
\text { maintaining the } \\
\text { diffusion gradient }\end{array}$ & $\begin{array}{l}\text { Very restricted and } \\
\text { almost negligible (in } \\
\text { the later phase of } \\
\text { experiment) due to } \\
\text { coagulated marrow, and } \\
\text { results in drug retention } \\
\text { inside the bone }\end{array}$ & $\begin{array}{l}\text { Partially effective as the } \\
\text { marrow impedes the } \\
\text { flow of culture media, } \\
\text { thereby the clearing rate } \\
\text { of dye is reduced }\end{array}$ \\
\hline
\end{tabular}

TABLE 2. Factors influencing release of model drug $(\mathrm{RhB})$ from the surface of TNTs/Ti wire implants directly inside the bone microenvironment ex-vivo. 